\title{
Confidence Measure for Temporal Registration of Recurrent Non-uniform Samples
}

\author{
Meghna Singh ${ }^{1}$, Mrinal Mandal ${ }^{1}$, and Anup Basu ${ }^{2}$ \\ ${ }^{1}$ Department of Electrical and Computer Engineering, \\ ${ }^{2}$ Department of Computing Science, \\ University of Alberta, Edmonton, AB, Canada \\ \{meghna, mandal\} @ece.ualberta.ca, \\ anup@cs . ualberta.ca
}

\begin{abstract}
Temporal registration refers to the methods used to align time varying sample sets with respect to each other. While reconstruction from a single sample set may generate aliasing, registration of multiple sample sets increases the effective sampling rate and therefore helps alleviate the problems created by low acquisition rates. However, since registration is mostly computed as an iterative best estimate, any error in registration translates directly into an increase in reconstruction error. In this paper we present a confidence measure based on local and global temporal registration errors, computed between sample sets, to determine if a given set of samples is suitable for inclusion in the reconstruction of a higher resolution temporal dataset. We also discuss implications of the non-uniform sampling theorem on the proposed confidence measure. Experimental results with real and synthetic data are provided to validate the proposed confidence measure.
\end{abstract}

Keywords: Recurrent non-uniform sampling, Temporal registration, Confidence measure.

\section{Introduction}

Temporal registration, which is the computation of a correspondence in time between two sequences, has lately received much attention from researchers in the context of spatio-temporal super-resolution videos [1-3][9]. Temporal registration between sequences becomes imperative when a signal is acquired at a rate much lower than the highest frequency of the signal. Since reconstruction from a single sample set leads to aliasing, one proposed solution to this problem is to acquire multiple low sampled sequences, and compute a temporal relation between them. Fusing multiple low sampled sequences improves the effective sampling rate for reconstruction purposes. Our past work [1-3] dealt with estimating an event dynamics model and using it to register two videos acquired at low sampling rates. The computed registration was then further used to generate a temporally super-resolved video. In [1] we showed that using the global event dynamics for local registration is a better approach than local linear interpolation [9]. 
A limitation with using temporal registration to generate super-resolved videos [1]-[3][9] is that the registration is based on optimizations, such as linear least squares or projection on to convex sets. Since no time-stamp information is available, these optimizations are only a best estimate of the actual registration. The reconstruction process depends greatly on the computed registration, hence it is better to leave out a sample set for which the registration might be incorrect, rather than including it for reconstruction. In this work, we develop a confidence measure which can be used to determine if a sample set should be included or excluded from the reconstruction process, in order to minimize the reconstruction error. The following assumptions are made in this work: (i) A signal is sampled at a frequency much lower than the Nyquist sampling rate (this happens often in MRI acquisitions of dynamic events and in video acquisitions of fast occurring events), (ii) multiple such sample sets are available, (iii) the temporal relation between these low sampled sets can be computed using methods described in [1], however, there is uncertainty associated with this computed relation. In this work, we develop a confidence measure to answer the following questions: (i) How reliable is the computed temporal relation between the sample sets, (ii) How can we compute a confidence measure to decide if a sample set should be included or excluded from the reconstruction process?

The rest of this paper is organized as follows. In Section 2, we review related work in temporal registration and recurrent non-uniform sampling. In Section 3, we present the proposed confidence measure and discuss the various factors that influence its computation. In Section 4, we present the experimental setup and results of the reconstruction algorithm based on the developed confidence measure. Lastly, we present the conclusions of our work in Section 5.

\section{Review of Related Work}

Reconstruction from multiple sample sets which are offset from each other by a known time interval has been studied in the past as the domain of recurrent nonuniform sampling and reconstruction [4-8]. The non-uniform sampling theorem from [7] states that " $a$ function $f(t)$, bandlimited to $-\Omega / 2 \leq \omega \leq \Omega / 2$, can be uniquely reconstructed from a set of samples which are non-uniformly spaced but satisfy the condition that there be precisely $N$ distinct samples to every interval of length NT, where $N$ is some finite integer." Reference [7] also provides an interpolation formula for reconstruction of the signal from its non-uniform samples provided the condition in the theorem above is satisfied. Other researchers $([5],[6],[8]$ and references therein) have developed simplifications to the interpolation, such as global polynomial fitting, trigonometric polynomials, moving least squares, radial basis functions and variational approach using splines. We use the reconstruction algorithm from [10] to evaluate our confidence metric. Feichtinger et al. [10] compute sampling atoms or synthesis functions using approximation operators such that every bandlimited function has a stable summed expansion of the type shown in Eq.1, where $e_{n}$ are the sampling atoms. Unlike uniform sampling functions the sampling atoms are not necessarily translations of a mother 
function and are computed using frame theory and adaptive weights to improve the numerical efficiency of the computation.

$$
f=\sum_{n \in Z} f\left(t_{n}\right) e_{n}
$$

Confidence measures have been proposed in a variety of fields. In signal processing and pattern recognition, confidence measures have been computed extensively for speech recognition [11][12] . These confidence measures are mostly based on the probability distributions of the likelihood functions of speech utterances, which are derived from Hidden Markov Models. Our proposed work is unique as it introduces the concept of a confidence measure in temporal registration and reconstruction from recurrent non-uniform samples. It also solves a real world problem faced in temporal super-resolution - determining the worthiness of a sample set.

\section{Proposed Confidence Metric}

Let the recurrent sample sets have a fixed sampling interval $T$ which is much lower than the Nyquist sampling interval, as shown in Fig.1. In Fig.1, we illustrate two sample sets, offset from each other with a time interval $t_{0} \cdot t_{0}$ is modeled as a discrete uniform distribution as all values within the finite time interval are equally possible. Most reconstruction algorithms assume that the value of $t_{0}$ is known a priori (i.e. it is a controlled variable) or its exact value can be computed. However, in temporal registration $t_{0}$ is the interval that we seek to compute, often with an unknown degree of inaccuracy.

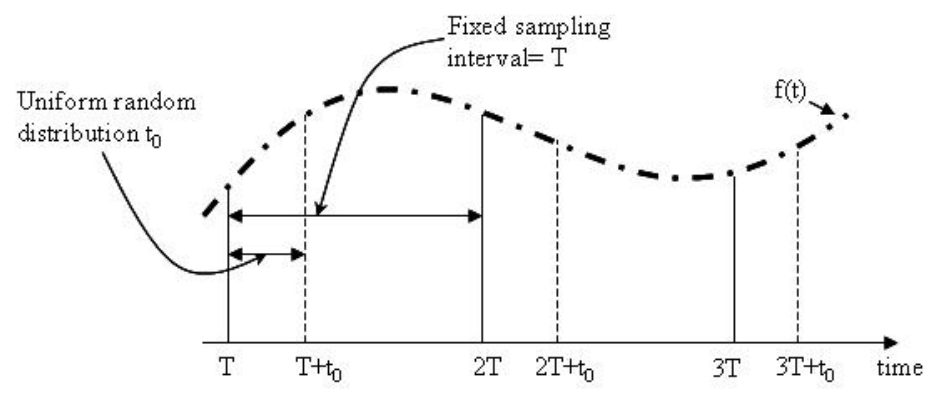

Fig. 1. Illustration of recurrent non-uniform sampling with two sample sets

In previous work [1-3] we computed $t_{0}$ by minimizing the registration error in the least square sense. In this work we express the registration error as an aggregate of local registration error and global registration error. Global registration error $\left(E_{g}\right)$ is the error computed from a one-to-one discrete sample correspondence. Local registration error $\left(E_{l}\right)$ on the other hand is computed by 
first constructing a continuous event model from all the discrete samples of a set, and then registering the event models on a sub-integer time level. Given two sample sets $f(k T)$ and $f\left(k T+t_{0}\right)$, as shown in Fig.1, the global registration error can be computed as follows:

$$
E_{g}=\arg \min _{t_{0}}\left(\sum_{k}\left\|f(k T)-f\left(k T+t_{0}\right)\right\|^{2}\right)
$$

Subsequent to computing the event models $c(t)$ and $c\left(t+t_{0}\right)$ by methods described in [1-3], the local registration error can be expressed as follows:

$$
E_{l}=\arg \min _{t_{0}}\left(\int\left\|c(t)-c\left(t+t_{0}\right)\right\|^{2} d t\right)
$$

The registration errors defined in Eq.2 and Eq.3 give a general idea of the confidence in the temporal registration. However, they do not relate to the confidence measure by a simple proportionality, i.e. a large global registration error does not imply a poor confidence in the registration. In fact, as we will demonstrate in our experiments, a large global registration error indicates a more uniform distribution of the sample sets and therefore a better reconstruction or a higher confidence. For now let us express the confidence measure as a weighted sum of $E_{g}$ and $E_{l}$ as follows:

$$
c(q, r)=w_{g} \times\left(E_{g}\right)^{q}+w_{l} \times\left(E_{l}\right)^{r}
$$

In Eq.4, $w_{g}$ and $w_{l}$ are weights assigned to the contribution of both the registration errors to the overall confidence measure. If no prior information is known about the registration then $w_{g}=w_{l}=0.5$. If prior information is known then the weights can be unequally assigned. The parameters $q$ and $r$ in Eq. 4 are defined as variables in the confidence measure as they can be used to incorporate non-linearity or to invert the proportionality of the registration errors. We present two hypotheses that are supported by experimental results shown in Fig. 2 and by the discussion that follows.

Hypothesis 1: $E_{g}$ is an indicator of $t_{0}$, and a value of $t_{0}$ which places the sample sets as far apart from each other as possible results in a better reconstruction, hence an increase in $E_{g}$ should have a positive effect on the confidence measure.

Hypothesis 2: $E_{l}$ is an indicator of the overall error in registration, and a large $E_{l}$ results in poorer reconstruction, hence an increase in $E_{l}$ should have a negative effect on the confidence measure.

Figure 2(a) shows the relationship between the reconstruction error and the global registration error. The reconstruction error reported in the figure is the mean of the SSE (sum of squared errors) of all the test cases, hence denoted as the MSE. It can be seen that $E_{g}$ demonstrates a linear relationship with the reconstruction error. As $E_{g}$ increases the reconstruction error decreases, i.e. the confidence measure which should be associated with $E_{g}$ should be in direct increasing proportion. Therefore ' $q$ ' in Eq.4 can be approximated to ' 1 '. We 
also fitted the MSE vs. $E_{g}$ curve with a quadratic function and it can be seen from Fig. 2(a) that a linear fit is a sufficiently good approximation of the curve. The same analysis is applied to the relationship between the reconstruction error and the local registration error $E_{l}$, Fig. 2(b). As $E_{l}$ increases, the reconstruction error increases. We invert the abscissa to plot MSE vs. $E_{l}^{-1}$, to define an inverse relationship between the confidence measure and $E_{l}$. Hence, ' $r$ ' in Eq.4 can be approximated to ' -1 '. The proposed confidence measure $\chi$ can therefore be expressed as follows:

$$
\chi=c(1,-1)=w_{g} \times\left(E_{g}\right)^{1}+w_{l} \times\left(E_{l}\right)^{-1}
$$

It would be interesting at this point to discuss the implications of the nonuniform sampling theorem to temporal registration. For there to be $N$ distinct samples to every interval of length $N T$, the average non-uniform sampling rate required is the same as the Nyquist rate. The farther the recurrent sample sets are spaced apart in time with respect to each other, the higher is the average sampling rate. This leads to the conclusion that for optimal reconstruction, $t_{0}$ should be close to mid-way between $k T$ and $(k+1) T$ samples in Fig.1. Such positioning of $t_{0}$ will lead to a large error in the global registration of two sequences, which supports Hypothesis 1.

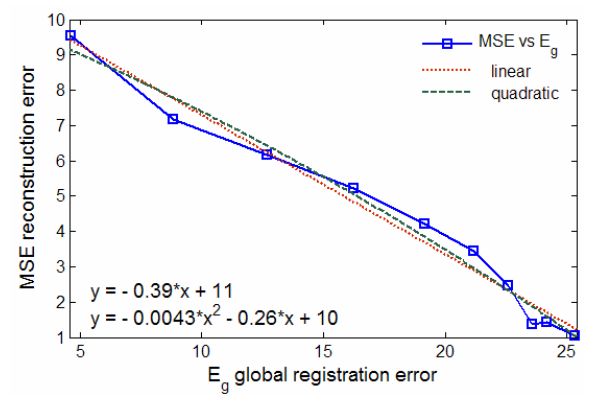

(a)

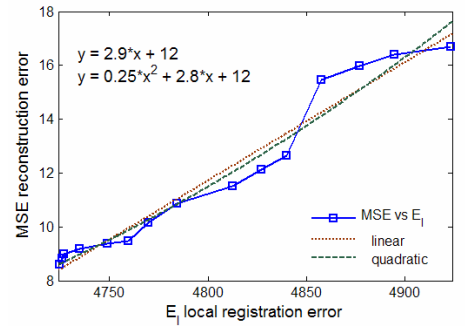

(b)

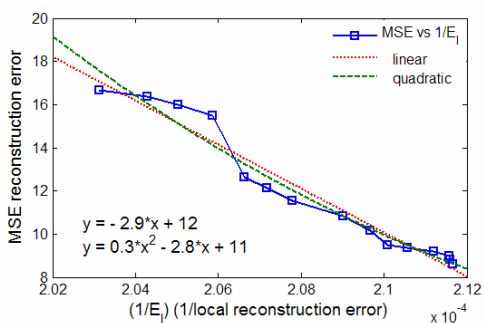

(c)

Fig. 2. (a) Relationship between mean square reconstruction error (MSE) and global registration error $E_{g}$, (b) Relationship between MSE and local registration error $E_{l}$, (c) Relationship between MSE and $\left(E_{l}^{-1}\right)$ 


\section{Experimental Setup and Results}

We tested the proposed confidence measure on both synthetic and real data. Samples of synthetic data and real test videos are available for viewing at: http: //www . ece.ualberta.ca/ meghna/Premi_webpage/PReMI_2007.html. Synthetic data was generated as a high resolution random signal which was band-limited to a user controlled frequency. This high resolution data was then sampled at a very low discrete sampling rate. For example, a $25 \mathrm{~Hz}$ band-limited signal was sampled at $2 \mathrm{~Hz}$. Multiple sample sets at a fixed low sample rate were also generated by initializing the starting point of the sample sets randomly with a uniform distribution. Temporal registration was then computed using methods described in [1]. However, since the samples in each set were limited, the computed registration was not accurate to a sub-frame level. These multiple sample sets were then iteratively fused together, one at a time, based on erroneous time stamp information. Reconstruction algorithm from [10] was used to reconstruct a signal from the fused samples. By adding a sample set at each iteration (see Fig.3 $(a-b))$, we were able to observe that the MSE falls as the number of sample sets increases. Also, as per our hypothesis, if the sample sets are such that the $t_{0}$ is small i.e. the sample sets are closely spaced, then the reconstruction error falls at a rate much slower than if the sample sets were further apart (Fig.3(c)). The relationships developed between $E_{g}, E_{l}$ and MSE for the synthetic test cases have already been presented in Fig.2.

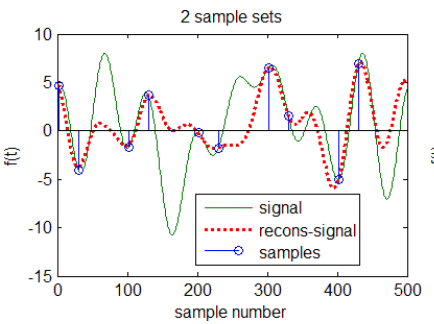

(a)

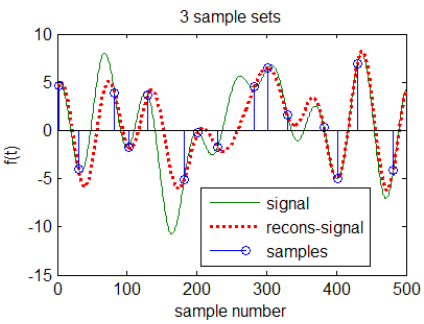

(b)

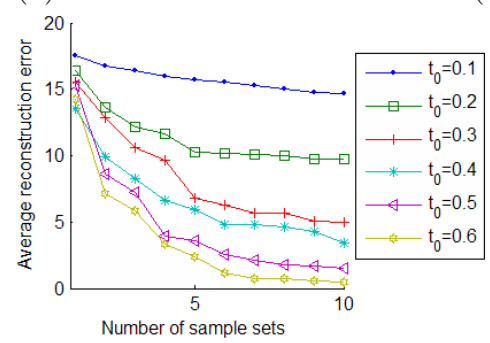

(c)

Fig. 3. (a) Reconstruction of the signal $f(t)$ with only two sample sets at very low sampling rate, (b) Reconstruction with three sample sets. (c) Illustration of decrease in reconstruction error with increase in $t_{0}$ (reported as a normalized number $[0,1]$ ). 


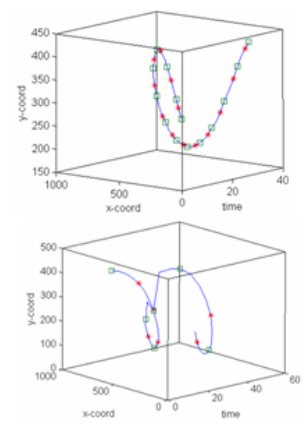

(a)

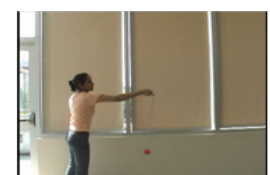

frame1

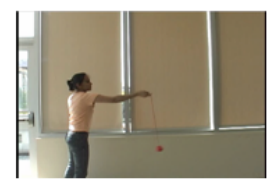

frame3

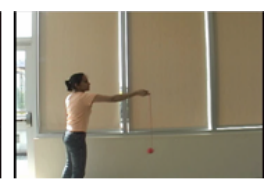

frame2

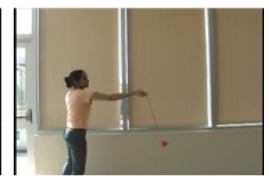

frame4

(b)

Fig. 4. (a) Sample trajectories from real data sequence, (b) Sample frames from real data sequence

For our real test cases, we used video sequences of an individual swinging a ball tied to the end of a string. The video sequences were captured at 30 frames per second and the trajectory of the ball was extracted via background subtraction techniques and motion tracking. This trajectory was then used as a high resolution signal which was further down-sampled at low sampling rates, as shown in Fig.4(a). An event model [1] was used to compute the temporal registration between the low sampled signals. In each experiment, we arbitrarily chose one sample set as the parent against whom two other recurrent sample sets were registered. Based on the local and global registration errors computed using Eq.2 and Eq.3 we determined the confidence measure as per Eq.5. The two confidence measures $\chi(1), \chi(2)$ for each recurrent set were compared to each

Table 1. Experimental results of confidence metric $\chi$ and reconstruction error (SSE)

\begin{tabular}{lllllllll}
\hline & $E_{g}(\mathbf{1})$ & $E_{l}(\mathbf{1})$ & $E_{g}(\mathbf{2})$ & $E_{l}(\mathbf{2})$ & $\chi(\mathbf{1})$ & $\chi(\mathbf{2})$ & $S S E \mathbf{( 1 )}$ & $S S E$ (2) \\
\hline \hline Seq1 & 44.0 & 230.3 & 35.6 & 596.7 & 1 & 0 & 1.3 & 2.6 \\
Seq2 & 15.7 & 49.8 & 4.0 & 8.9 & 1 & 0 & 4.0 & 13.0 \\
Seq3 & 22.5 & 374.0 & 16.8 & 212.4 & 1 & 0 & 205.4 & 355.3 \\
Seq4 & 97.2 & 2959.0 & 55.1 & 776.0 & 1 & 0 & 2.4 & 40.3 \\
Seq5 & 26.5 & 22.8 & 8.7 & 20.6 & 1 & 0 & 47.3 & 68.6 \\
Seq6 & 446.0 & 41.1 & 652.0 & 22.3 & 0 & 1 & 1700 & 4.2 \\
Seq7 & 234.0 & 90.3 & 652.0 & 22.3 & 0 & 1 & 81200 & 42000 \\
Seq8 & 129.0 & 4.6 & 364.0 & 321.0 & 0 & 1 & 20.0 & 4.0 \\
Seq9 & 43.7 & 7081.5 & 19.7 & 2228.7 & 1 & 0 & 8.9 & 16.1 \\
Seq10 & 7.8 & 443.4 & 28.9 & 3687.7 & 0 & 1 & 10.9 & 10.3 \\
Seq11 & 21.6 & 7409.3 & 16.8 & 5970.7 & 1 & 0 & 8.7 & 9.3 \\
Seq12 & 28.9 & 5823.3 & 22.3 & 3121.5 & 1 & 0 & 7.9 & 10.7 \\
Seq13 & 16.3 & 2387.7 & 17.4 & 2590.6 & 0 & 1 & 9.7 & 9.3 \\
Seq14 & 4.6 & 169.5 & 30.3 & 6190.9 & 0 & 1 & 15.6 & 9.9 \\
\hline
\end{tabular}


other. If $\chi(1)>\chi(2)$, then $\chi(1)$ was given an absolute value of 1 , else it was set to 0 . If more than two sample sets are being compared then a normalized value can be assigned to the confidence measure. Table 1 shows the local and global registration errors for a parent set and two recurrent sets, for 14 different signals including both synthetic and real data (real data is from seq6-8). A confidence metric of 1 indicates that the corresponding recurrent set is a better candidate for reconstruction, as proven by the corresponding computed reconstruction error. It can be seen that the proposed confidence metric is a suitable indicator of the reconstruction error.

\section{Conclusion}

In this work, we presented a confidence measure to determine the suitability of recurrent sample sets for reconstruction purposes based on the computed temporal registration errors. Experiments with real and synthetic data were conducted, and the results support the proposed confidence measure. The confidence measure is able to successfully indicate the sample sets which would lead to a higher error if included in the reconstruction process. As part of future work we plan to use this confidence measure with recurrent sample sets of MRI data.

\section{References}

1. Singh, M., Basu, A., Mandal, M.: Event Dynamics based Temporal Registration. IEEE Trans. on Multimedia 9(5), 1004-1015 (2007)

2. Singh, M., Thompson, R., Basu, A., Rieger, J., Mandal, M.: Image based Temporal Registration of MRI Data for Medical Visualization. In: Intl. Conf. on Image Processing ICIP, October 8-11, 2006, pp. 1169-1172

3. Singh, M., Basu, A., Mandal, M.: Temporal Alignment of Time Varying MRI Datasets for High Resolution Medical Visualization. In: Intl. Symposium on Visual Computing ISVC, pp. 222-231 (2006)

4. Kim, S.P., Bose, N.K.: Reconstruction of 2D bandlimited discrete signals from nonuniform samples. IEE Proceedings 137(3) (June 1990)

5. Arigovindan, M., Suhling, M., Hunziker, P., Unser, M.: Variational Image Reconstruction from Arbitrarily Spaced Samples: A Fast Multiresolution Spline Solution. IEEE Trans. on Image Processing 14(4) (April 2005)

6. Micchelli, C.A.: Interpolation of scattered data: distance matrices and conditionally positive definite functions, Constructive Approximation 2 (1986)

7. Freeman, H.: Discrete-Time Systems. John Wiley and Sons Inc, Chichester (1965)

8. Marvasti, F.: Nonuniform Sampling Theory and Practice. Kluwer Academic, New York (2001)

9. Shechtman, E., Caspi, Y., Irani, M.: Space-time super-resolution. IEEE Trans. on Pattern Analysis and Machine Intelligence 27(4), 531-545 (2005)

10. Feichtinger, H.G., Werther, T.: Improved Locality for Irregular Sampling Algorithms. In: Proc. ICASSP (1999)

11. Mortensen, E.N., Barrett, W.A.: A Confidence Measure for Boundary Detection and Object Selection. In: Proc. CVPR (2001)

12. Moreau, N., Jouvet, D.: Use of a confidence measure based on frame level likelihood ratios for the rejection of incorrect data. In: Proc. Eurospeech (1999) 